\title{
Templated growth of diamond optical resonators via plasma-enhanced chemical vapor deposition
}

\section{Citation}

Zhang, X., and E. L. Hu. 2016. "Templated Growth of Diamond Optical Resonators via PlasmaEnhanced Chemical Vapor Deposition." Applied Physics Letters 109 (8) (August 22): 081101. doi:10.1063/1.4961536.

\section{Published Version}

doi:10.1063/1.4961536

\section{Permanent link}

http://nrs.harvard.edu/urn-3:HUL.InstRepos:33980565

\section{Terms of Use}

This article was downloaded from Harvard University's DASH repository, and is made available under the terms and conditions applicable to Other Posted Material, as set forth at http:// nrs.harvard.edu/urn-3:HUL.InstRepos:dash.current.terms-of-use\#LAA

\section{Share Your Story}

The Harvard community has made this article openly available.

Please share how this access benefits you. Submit a story.

Accessibility 


\title{
Templated growth of diamond optical resonators via plasma-enhanced chemical vapor deposition
}

\author{
X. Zhang ${ }^{\text {a) }}$ and E. L. Hu \\ John A. Paulson School of Engineering and Applied Sciences, Harvard University, 29 Oxford Street, \\ Cambridge, Massachusetts 02138, USA
}

(Received 28 April 2016; accepted 11 August 2016; published online 22 August 2016)

\begin{abstract}
We utilize plasma-enhanced chemical vapor deposition through a patterned silica mask for templated diamond growth to create optical resonators. The pyramid-shaped structures have quality factors $Q$ up to 600, measured using confocal photoluminescence spectroscopy, and mode volumes $V$ as small as $2.5(\lambda / n)^{3}$ for resonances at wavelengths $\lambda$ between 550 and $650 \mathrm{~nm}$, and refractive index $n$, obtained using finite-difference time-domain simulations. Bright luminescence from nitrogen-vacancy and silicon-vacancy centers in the grown diamond is observed. The resonator design and fabrication technique obviates any etching of diamond, which preserves emitter properties in a pristine host lattice. Published by AIP Publishing. [http://dx.doi.org/10.1063/1.4961536]
\end{abstract}

Color centers in diamond, ${ }^{1}$ including the nitrogen-vacancy ${ }^{2}$ $(\mathrm{NV})$ and silicon-vacancy ${ }^{3}(\mathrm{SiV})$ centers, have diverse applications for nanoscale sensing ${ }^{4}$ and quantum information science. ${ }^{5}$ Diamond photonic structures assist in the manipulation and optical readout of the color centers' electron spin state by increasing collection efficiency ${ }^{6}$ and emission rate using the Purcell effect. ${ }^{7-9}$ Such micro- and nanostructures ${ }^{10}$ have typically been created using traditional, top-down fabrication techniques, including reactive ion etching (RIE) and focused ion beam milling. However, it is useful also to explore bottom-up approaches in which photonic nanostructures are formed directly through templated growth of diamond by chemical vapor deposition (CVD). ${ }^{11}$ This allows incorporation of color centers directly during growth, ${ }^{11-14}$ and minimizes the impact of energetic ions or electron beams that can incur damage ${ }^{15}$ to color centers, which are highly sensitive to their local environment. While templated growth has been previously reported in heteroepitaxial structures ${ }^{14,16}$ or with nano- and polycrystalline diamond seeding, ${ }^{12,17}$ the application described here poses more stringent demands on controlling the shape and material quality of the grown diamond. In this work, we use templated epitaxial growth of diamond by CVD, combining control over the morphology of the resulting structures and in situ incorporation of color centers, to create pyramid-shaped optical resonators for emission enhancement of the embedded emitters.

Pyramid structures can support whispering gallery-type resonances ${ }^{18}$ that confine light from total internal reflection similar to microdisk resonators. ${ }^{19} \mathrm{GaAs}$ and $\mathrm{GaN}$ pyramids have also been used for dielectric ${ }^{20}$ and plasmonic ${ }^{21}$ cavities, respectively, using the appropriate fabrication techniques for each material system. We performed 3D finite-difference time-domain (FDTD) simulations (Lumerical Solutions) of square pyramid structures (Fig. 1) to understand the spatial field distribution of the resonances and to optimize resonator design. A pair of dipole sources with a spectral profile similar to that of the NV was positioned within the pyramid structures to emulate embedded color centers. Time-varying

${ }^{\text {a)} E l e c t r o n i c ~ m a i l: ~ x z h a n g 4 @ s e a s . h a r v a r d . e d u ~}$ electric fields within the simulation region were recorded and used to compute the quality factor $Q$ and mode volume ${ }^{22} \mathrm{~V}$ for the resonances. Emission enhancement in optical resonators due to the Purcell effect ${ }^{23}$ is proportional to $Q / V$, which serves as a figure of merit for the design. For $s=1.5 \mu \mathrm{m}$, we find a resonance at $\lambda_{0}=621 \mathrm{~nm}$, which has $Q=515$ and electric field profiles (Fig. 1(c)) from the simulations. We found that a supporting post with height $h=300 \mathrm{~nm}$ and diameter $d=300 \mathrm{~nm}$ is sufficient to preserve high- $Q$ resonances (Fig. 2(a)). The electric field profile recorded at the top of the pyramid was used for a far-field projection, which suggests improved collection efficiency, similar to solid immersion lens-like structures in Ref. 13. Larger pyramids have higher $Q$, but correspondingly larger $V$, so that $Q / V$ is maximized for the $s=1.5 \mu \mathrm{m}$ structure (Fig. 2(b)).

Our general technique for templated growth uses patterned thin films of $\mathrm{SiO}_{2}$ placed directly onto a single-crystal diamond substrate. Subsequent plasma-enhanced CVD (PECVD) of diamond ${ }^{24}$ through the growth masks produces the desired photonic structures. When the mask is thicker than the deposited diamond, the constrained structures adopt the shape of the patterned template. For growth that exceeds the mask thickness, the shape of the overgrown region is determined by the relative growth rates along different crystal planes. In those structures, the smaller conformal region below forms a post which supports the structure above (Fig. 1(b)). We optimized growth conditions where the relative growth rate of the $\{100\}$ faces $\left(V_{100}\right)$ is larger than that of the $\{111\}$ faces $\left(V_{111}\right)$ to form pyramidal top structures. When $V_{100}=V_{111} \sqrt{3}$, the $\{100\}$ faces are extinguished and the remaining $\{111\}$ faces form an octahedron. ${ }^{25}$ Growth from a bulk planar substrate through a silica mask forms one half of an octahedron, i.e., a square pyramid. We use a wet etch following the templated growth to remove the silica mask, resulting in an undercut structure which provides optical isolation from the bulk substrate.

For the growth substrate, we used an HPHT diamond (Element Six), which was cleaned using a refluxing acid mixture (1:1:1 nitric:perchloric:sulfuric acids) for $3 \mathrm{~h}$. It was then rinsed in boiling piranha mixture (3:1 sulfuric acid:hydrogen peroxide), then rinsed and sonicated in solvent and 
(a)

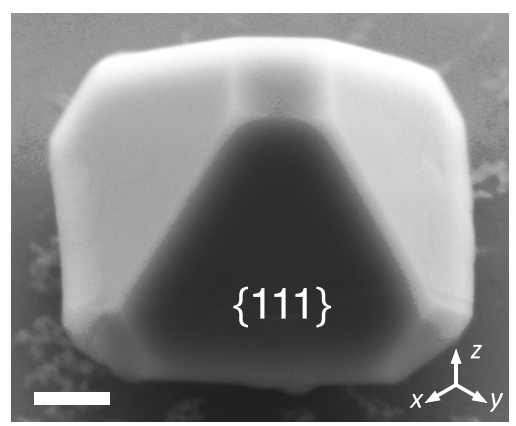

(b)

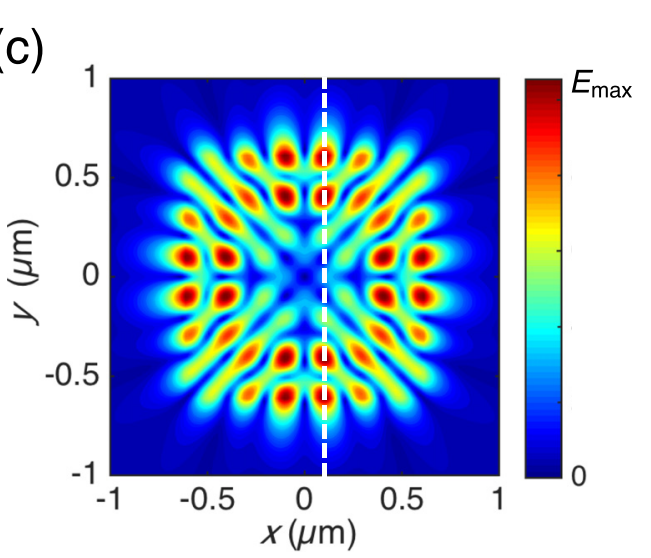

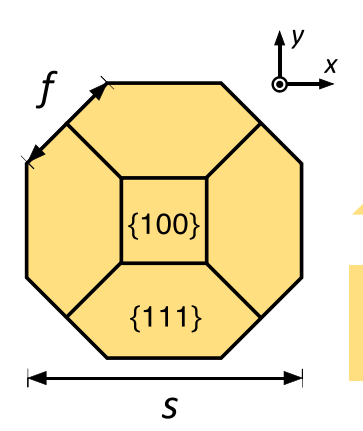

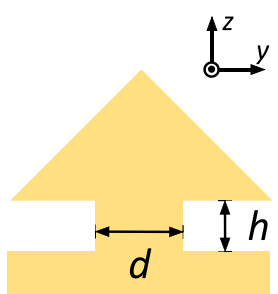

Substrate

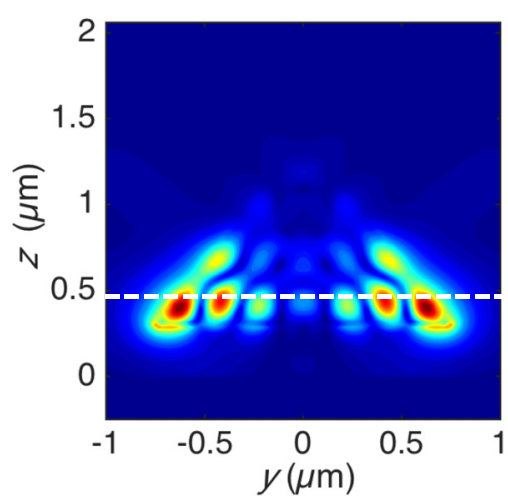

FIG. 1. Scanning electron micrograph of a pyramid resonator. Scale bar indicates $250 \mathrm{~nm}$ (a). Schematic showing resonator geometry and simulation parameters (b). Electric field profiles for a resonator with $s=1.5 \mu \mathrm{m}$ on resonance at $621 \mathrm{~nm}$ (c). Dashed lines indicate positions where the field profiles are recorded. dried. Next, we deposited $15 \mathrm{~nm}$ of Ti using electron-beam evaporation (Denton Explorer) and patterned a $300 \mathrm{~nm}$-thick silica film on top using electron-beam lithography (EBL). The mask is deposited using a spin-on, negative-tone EBL resist (Dow Corning FOx-16) containing hydrogen silsesquioxane. Regions of the resist exposed during EBL forms the silica-based mask, while the unexposed areas, after development in tetramethylammonium hydroxide, form the apertures (diameters ranging from 200-400 nm) through which the posts and pyramid structures are formed during diamond CVD. Next, we removed the exposed Ti using RIE with an Ar-Cl plasma (Unaxis Shuttleline). We carried out the diamond growth using microwave PECVD (Seki Technotron AX5010-INT) in a hydrogen plasma at $1000 \mathrm{~W}$ power, 40 Torr pressure, with $400: 4 \mathrm{sccm}$ of $\mathrm{H}_{2}: \mathrm{CH}_{4}$ gas for 90 min with a substrate temperature of $760{ }^{\circ} \mathrm{C}$ measured using a pyrometer. Finally, the patterned silica mask is removed using hydrofluoric acid to create undercut pyramid structures.

A typical pyramid resonator is shown in the scanning electron micrograph in Fig. 1(a). Arrays of the fabricated structures are nearly identical and growth defects (e.g., twinning) were minimized through optimizing the CVD growth conditions. The remaining $\{100\}$ faces of the pyramidal structures suggest that $1 / \sqrt{3}<V_{100} / V_{111}<\sqrt{3}$, but the presence of the $\{100\}$ faces is not expected to adversely affect the $Q$ of the resonator (Fig. 2(a)). Photoluminescence (PL) and Raman spectra from the substrate and resonators were collected using $532 \mathrm{~nm}$ laser excitation with a confocal photoluminescence (PL) microscope (Horiba Jobin Yvon) using a $0.95 \mathrm{NA}$ objective at $100 \times$ magnification. A Voigt fit for the first-order Raman line gives $1332.43 \pm 0.05 \mathrm{~cm}^{-1}$ for the diamond substrate with FWHM of $2.44 \pm 0.02 \mathrm{~cm}^{-1}$, which is comparable with the values found for the grown resonators, with center at $1332.59 \pm 0.13 \mathrm{~cm}^{-1}$ and FWHM of $2.60 \pm 0.11 \mathrm{~cm}^{-1}$, indicating high-quality material from the growth. ${ }^{26}$ Using the PL data from a sample of 20 resonators with $s \approx 1.1$ and $1.6 \mu \mathrm{m}$, we see that the Lorentzian-fitted values for $Q$ of cavity resonances agree well with simulation results (Fig. 2(b)). The improvement in collection efficiency is shown by an increase in the PL count rate from the pyramid structures versus thin film regions, both of which are grown on the same sample during the same CVD process (Fig. 3(a)). The thin film growth occurs on areas of the substrate that are not patterned with silica. We observe from the pyramids that the intensity of the diamond Raman line is 1.6 times greater and more than double for the $\mathrm{NV}^{-}$and $\mathrm{SiV}^{-}$ zero phonon lines (ZPL). However, proximity to the silica mask can affect Si incorporation, so the Raman signal provides the best comparison for the collection efficiency. The same increase is observed for multiple pyramids and thin film regions, including the original (pre-growth) diamond substrate.

For a grown pyramid resonator with $s=1.5 \mu \mathrm{m}$ and $f=300 \mathrm{~nm}$, a Lorentzian fit for the resonance gives $Q=600$ at $616.8 \mathrm{~nm}$ (Fig. 3(a)), which agrees with simulation results with $Q=620$ at $621 \mathrm{~nm}$. We observed bright luminescence from neutral and negatively charged $\mathrm{NV}$ and $\mathrm{SiV}$ centers from the resonators, indicating the incorporation of color centers during growth. The $\mathrm{Si}$ in the $\mathrm{SiV}$ centers originated from the silica-based growth template, which could be eliminated or reduced by choosing a different mask material (e.g., aluminum oxide) in order to produce only NV or single $\mathrm{SiV}$ centers during growth. We also demonstrated control over the cavity resonance by changing the resonator size. For resonators with $s \sim 1 \mu \mathrm{m}$, the resonant wavelengths increase 
(a)

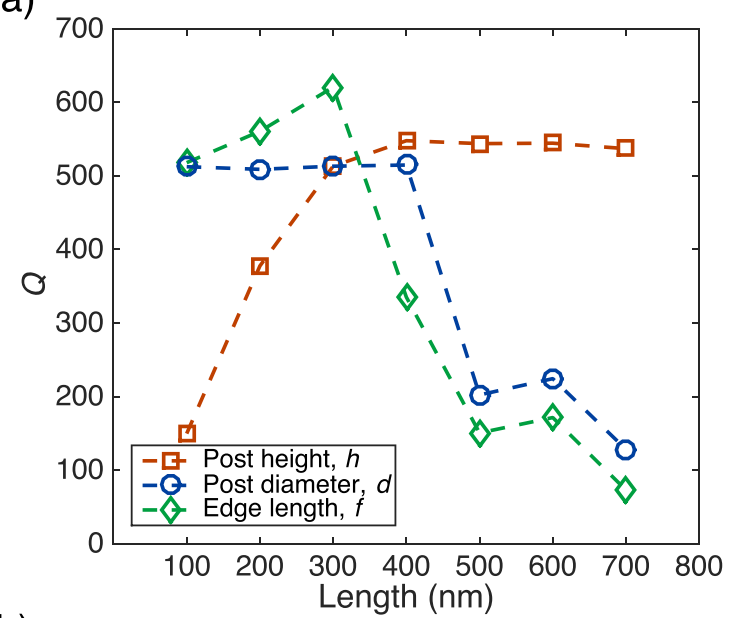

(b)

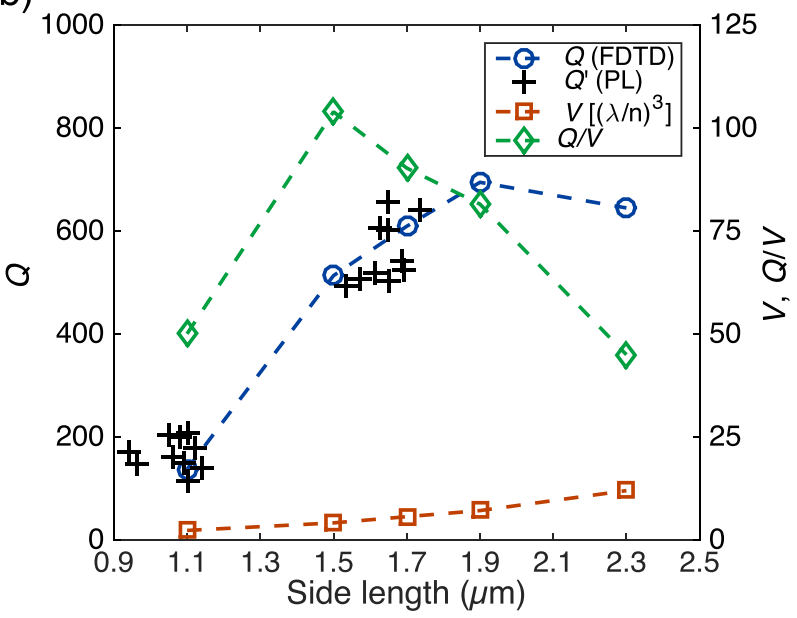

FIG. 2. FDTD simulation results for $Q$ with $s=1.5 \mu \mathrm{m}$ and different values of post height, diameter, and edge length (a). Simulation results for $Q, V$ and fitted values for $Q$ from PL measurements (crosses) for different pyramid side lengths (b).

for increasing $s$, where the fitted values for $\lambda_{0}$ and $Q$ agree with simulated results (Fig. 3(b)). Furthermore, for the structure with $s=1.05 \mu \mathrm{m}$, we found the simulated $V=2.5(\lambda / n)^{3}$, which is smaller than that for existing diamond microdisk resonators ${ }^{27}$ where $V \sim 10(\lambda / n)^{3}$ but also larger $Q=2200$.

In summary, we designed and utilized templated growth of diamond via PECVD to create pyramid resonators with measured $Q$ up to 600. By changing the resonator dimensions, we also controllably changed the resonant wavelengths. This allows these structures to be used for selective emission enhancement of the $\mathrm{NV}$ and $\mathrm{SiV}$ centers incorporated during growth. We used high-quality overgrowth of single-crystal diamond and control over its shape and dimensions to create structures with deterministic resonances and increased measured luminescence from incorporated color centers. This demonstrates the promise for bottom-up approaches for creating diamond photonic nanostructures. We expect that this fabrication technique can be further developed to spatially localize NVs (e.g., via delta-doping ${ }^{28}$ ) within a single photonic structure or in large numbers of highly uniform diamond nano- and microstructures. ${ }^{29}$ Furthermore, modifying the composition and features of the growth mask would allow creating other diamond nanostructures hosting color centers.

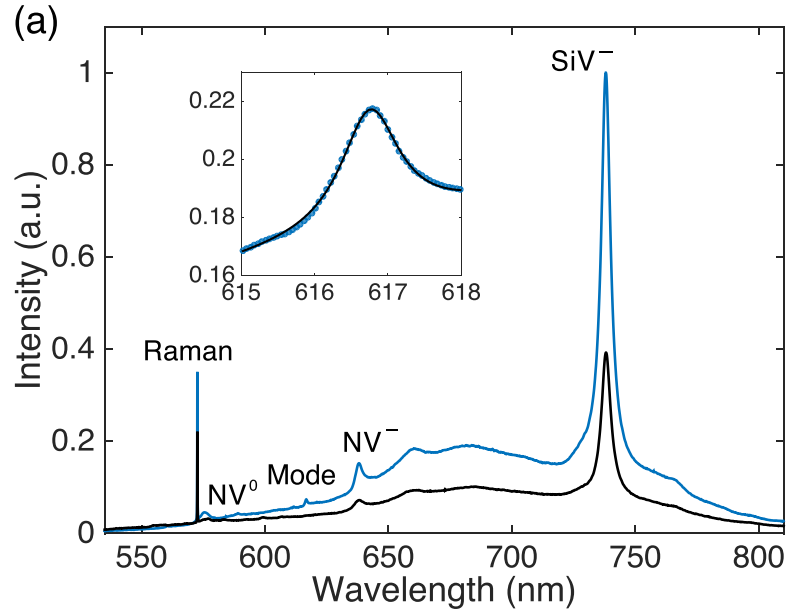

(b)

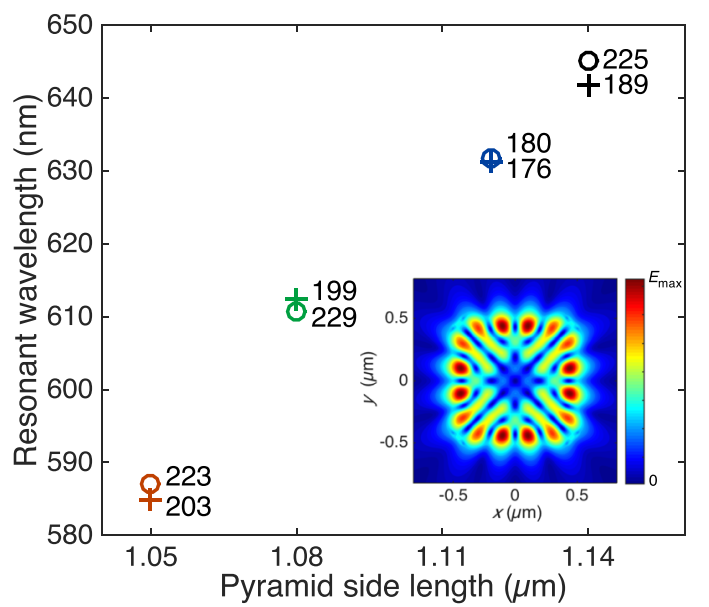

FIG. 3. PL spectrum of an $s=1.5 \mu \mathrm{m}$ pyramid (blue) and thin film (black) showing the diamond Raman line, NV, SiV luminescence, and the resonator mode detailed in inset (a). FDTD simulation (circles) and measured (crosses) resonant wavelengths labeled with fitted values for $Q$ for $s \sim 1 \mu \mathrm{m}$ pyramids with the electric field profile for the resonant mode in inset (b).

This work was performed in part at the Harvard University Center for Nanoscale Systems (CNS), a member of the National Nanotechnology Coordinated Infrastructure Network (NNCI), which is supported by the National Science Foundation (NSF) under NSF Award No. 1541959. This work was supported by the NSF STC Center for Integrated Quantum Materials, NSF Grant DMR-1231319. The authors thank D. Bracher for helpful discussions.

${ }^{1}$ A. M. Zaitsev, Optical Properties of Diamond (Springer, 2013).

${ }^{2}$ M. W. Doherty, N. B. Manson, P. Delaney, F. Jelezko, J. Wrachtrup, and L. C. L. Hollenberg, Phys. Rep. 528, 1 (2013).

${ }^{3}$ C. Hepp, T. Müller, V. Waselowski, J. N. Becker, B. Pingault, H. Sternschulte, D. Steinmüller-Nethl, A. Gali, J. R. Maze, M. Atatüre, and C. Becher, Phys. Rev. Lett. 112, 036405 (2014).

${ }^{4}$ R. Schirhagl, K. Chang, M. Loretz, and C. L. Degen, Annu. Rev. Phys. Chem. 65, 83 (2014).

${ }^{5}$ S. Prawer and I. Aharonovich, Quantum Information Processing with Diamond: Principles and Applications (Elsevier, 2014).

${ }^{6}$ L. Marseglia, J. P. Hadden, A. C. Stanley-Clarke, J. P. Harrison, B. Patton, Y.-L. D. Ho, B. Naydenov, F. Jelezko, J. Meijer, P. R. Dolan, J. M. Smith, J. G. Rarity, and J. L. O’Brien, Appl. Phys. Lett. 98, 133107 (2011).

${ }^{7}$ A. Faraon, P. E. Barclay, C. Santori, K.-M. C. Fu, and R. G. Beausoleil, Nat. Photon. 5, 301 (2011).

${ }^{8}$ J. C. Lee, D. O. Bracher, S. Cui, K. Ohno, C. A. McLellan, X. Zhang, P. Andrich, B. Alemán, K. J. Russell, A. P. Magyar, I. Aharonovich, A. Bleszynski Jayich, D. Awschalom, and E. L. Hu, Appl. Phys. Lett. 105, 261101 (2014). 
${ }^{9}$ L. Li, T. Schröder, E. H. Chen, M. Walsh, I. Bayn, J. Goldstein, O. Gaathon, M. E. Trusheim, M. Lu, J. Mower, M. Cotlet, M. L. Markham, D. J. Twitchen, and D. Englund, Nat. Commun. 6 (2015).

${ }^{10}$ T. Schröder, S. L. Mouradian, J. Zheng, M. E. Trusheim, M. Walsh, E. H. Chen, L. Li, I. Bayn, and D. Englund, J. Opt. Soc. Am. B 33, B65 (2016).

${ }^{11}$ I. Aharonovich, J. C. Lee, A. P. Magyar, D. O. Bracher, and E. L. Hu, Laser Photon. Rev. 7, L61 (2013).

${ }^{12}$ D. Sovyk, V. Ralchenko, M. Komlenok, A. A. Khomich, V. Shershulin, V. Vorobyov, I. Vlasov, V. Konov, and A. Akimov, Appl. Phys. A 118, 17 (2015).

${ }^{13}$ S. Furuyama, K. Tahara, T. Iwasaki, M. Shimizu, J. Yaita, M. Kondo, T. Kodera, and M. Hatano, Appl. Phys. Lett. 107, 163102 (2015).

${ }^{14}$ C. Arend, P. Appel, J. N. Becker, M. Schmidt, M. Fischer, S. Gsell, M. Schreck, C. Becher, P. Maletinsky, and E. Neu, Appl. Phys. Lett. 108, 063111 (2016).

${ }^{15}$ S. Cui, A. S. Greenspon, K. Ohno, B. A. Myers, A. C. B. Jayich, D. D. Awschalom, and E. L. Hu, Nano Lett. 15, 2887 (2015).

${ }^{16}$ Y. Ando, J. Kuwabara, K. Suzuki, and A. Sawabe, Diamond Relat. Mater. 13, 1975 (2004).

${ }^{17}$ H. Masuda, T. Yanagishita, K. Yasui, K. Nishio, I. Yagi, T. N. Rao, and A. Fujishima, Adv. Mater. 13, 247 (2001).

${ }^{18}$ W.-H. Guo, Y.-Z. Huang, Q.-Y. Lu, and L.-J. Yu, IEEE J. Quantum Electron. 39, 1106 (2003).
${ }^{19}$ B. Khanaliloo, M. Mitchell, A. C. Hryciw, and P. E. Barclay, Nano Lett. 15, 5131 (2015).

${ }^{20}$ F. M. Weber, M. Karl, J. Lupaca-Schomber, W. Löffler, S. Li, T. Passow, J. Hawecker, D. Gerthsen, H. Kalt, and M. Hetterich, Appl. Phys. Lett. 90, 161104 (2007).

${ }^{21}$ S.-H. Gong, J.-H. Kim, Y.-H. Ko, C. Rodriguez, J. Shin, Y.-H. Lee, L. S. Dang, X. Zhang, and Y.-H. Cho, Proc. Natl. Acad. Sci. U. S. A. 112, 5280 (2015).

${ }^{22}$ R. Coccioli, M. Boroditsky, K. Kim, Y. Rahmat-Samii, and E. Yablonovitch, IEE Proc.-Optoelectron. 145, 391 (1998).

${ }^{23}$ E. M. Purcell, Phys. Rev. 69, 37 (1946).

${ }^{24}$ S. Koizumi, C. Nebel, and M. Nesladek, Physics and Applications of CVD Diamond (Wiley, 2008).

${ }^{25}$ C. Wild, R. Kohl, N. Herres, W. Müller-Sebert, and P. Koidl, Diamond Relat. Mater. 3, 373 (1994).

${ }^{26}$ S. Prawer and R. J. Nemanich, Philos. Trans. R. Soc. A 362, 2537 (2004).

${ }^{27}$ J. C. Lee, I. Aharonovich, A. P. Magyar, F. Rol, and E. L. Hu, Opt. Express 20, 8891 (2012).

${ }^{28}$ K. Ohno, F. J. Heremans, L. C. Bassett, B. A. Myers, D. M. Toyli, A. C. B. Jayich, C. J. Palmstrom, and D. D. Awschalom, Appl. Phys. Lett. 101, 082413 (2012).

${ }^{29}$ P. Andrich, B. J. Alemán, J. C. Lee, K. Ohno, C. F. de las Casas, F. J. Heremans, E. L. Hu, and D. D. Awschalom, Nano Lett. 14, 4959 (2014). 